\title{
Breast cancer resistance protein (BCRP)-containing circulating microvesicles contribute to chemoresistance in breast cancer
}

\author{
YUN CHEN $^{1 *}$, LINJUN WANG $^{2^{*}}$, YIFEI ZHU ${ }^{1 *}$, ZHEN CHEN $^{1}$, XIAOWEI QI ${ }^{3}$, \\ LINFANG JIN $^{3}$, JIAN JIN ${ }^{1}$, DONG HUA ${ }^{2,3}$ and XIN MA ${ }^{1}$ \\ ${ }^{1}$ School of Pharmaceutical Sciences, Jiangnan University, Wuxi, Jiangsu 214122; \\ Departments of ${ }^{2}$ Medical Oncology and ${ }^{3}$ Pathology, Affiliated Hospital of Jiangnan University, \\ Wuxi, Jiangsu 204062, P.R. China
}

Received November 3, 2014; Accepted September 17, 2015

DOI: $10.3892 / 01.2015 .3806$

\begin{abstract}
At present, one of the major problems of cancer therapy is drug resistance. Breast cancer resistance protein (BCRP), a marker of the multidrug-resistant phenotype, affects drug absorption, distribution, metabolism, and excretion in normal tissues. Meanwhile, extracellular vesicles (EVs) have attracted increasing attention as a medium of cell-to-cell communication. However, the association between BCRP and circulating EVs remains unclear. The present study demonstrated that patients who did not respond or had progressive/ stable disease following chemotherapy had markedly higher BCRP levels compared to those that did not receive chemotherapy. Moreover, BCRP was upregulated at the mRNA and protein levels in tumor-derived circulating EVs from patients with a poor response to chemotherapy. Interestingly, the results also demonstrated that BCRP was co-expressed with MUC1, which is frequently expressed in breast cancer and is exported via EVs, and both BCRP and MUC1 were up-regulated after chemotherapy. In conclusion, the present study indicates that tumor-derived circulating EVs that carry BCRP may serve as a predictive biomarker of the response to chemotherapy for breast cancer. In addition, the results provide a window for individualized treatment to overcome resistance to chemotherapeutic drugs.
\end{abstract}

Correspondence to: Mr. Xin Ma, School of Pharmaceutical Sciences, Jiangnan University, 1800 Lihu Road, Wuxi, Jiangsu 214122, P.R. China

E-mail: maxin@jiangnan.edu.cn

Mr. Dong Hua, Department of Medical Oncology, Affiliated Hospital of Jiangnan University, 200 Huine Road, Wuxi, Jiangsu 204062, P.R. China

E-mail: huadong@csco.org.cn

*Contributed equally

Key words: breast cancer resistance protein, chemotherapeutic resistance, extracellular vesicles, mucin 1 , predictive biomarker

\section{Introduction}

Resistance to chemotherapy is a major problem facing current cancer therapy (1). Scientists continue to search for the mechanisms underlying drug resistance; meanwhile, identifying improved predictive biomarkers for responsiveness to chemotherapy remains important, since the traditional tumor markers such as MUC1 antigen (CA15.3) do not accurately reflect the actual effect of cancer treatment (2). It is necessary to define specific characteristics that provide the possibility of optimizing individual treatment (3), and in this era of high-throughput methods, a deluge of novel biomarkers have been reported for prognostic and predictive purposes. However, of these, only a few have been incorporated into clinical practice (3).

Intercellular communication is a hallmark of multicellular organisms and may be mediated through direct cell-cell contact or the transfer of secreted molecules (4). In the last 2 decades, a third mechanism of intercellular communication has emerged that involves the transfer of extracellular vesicles (EVs) shed from the cell plasma membrane (4). EVs are generally referred to as microvesicles, exosomes, shedding vesicles, or microparticles, among others (5-8).

Numerous diverse biological functions have been attributed to EVs (9), and it is now commonly accepted that exosomes and microvesicles are important vehicles of intercellular communication between cells locally and at a distance (4). Tumor cells, in addition to other cells in the tumor microenvironment, also release EVs, and there is evidence that they contribute to tumor progression by promoting angiogenesis and metastasis (4). Our previous study demonstrated that circulating EVs containing the $\mathrm{Ca}^{2+}$ - permeable channel TrpC5 transfer chemoresistance to previously non-chemoresistant recipient cells (10). This study led to the hypothesis that EVs may serve an essential role in clinical chemoresistance, therefore the present study aimed to investigate the association between EVs and drug resistance.

$\mathrm{ABCG} 2 /$ breast cancer resistance protein (BCRP) is a member of the adenosine triphosphate-binding cassette (ABC) transporter protein family. It is referred to as a 'half-type' $\mathrm{ABC}$ transporter, functions as a homodimer, and transports anticancer agents such as irinotecan out of cells (11). It has been demonstrated that elevated BCRP levels in vitro result 
in resistance to anticancer drugs, including topotecan and irinotecan, however the underlying mechanism(s) require further study (12), particularly whether BCRP acts via circulating EVs. If so, BCRP-containing EVs may have clinical applications. To investigate these possibilities, the present study investigated the association between BCRP and circulating EVs, carefully comparing patients demonstrating a poor response to chemotherapy with those without chemotherapy. The results indicated that there is a role of BCRP-containing EVs in clinical prognosis.

\section{Materials and methods}

Patient tumor specimens and peripheral blood samples. Tumor specimens for assessing the response to chemotherapy prior to surgery: Patients were recruited at the Affiliated Hospital of Jiangnan University (Wuxi, China) between 2010 and 2012. The anthracycline-taxane-based neoadjuvant chemotherapy regimen TEC (docetaxel, epirubicin, and cyclophosphamide) was used with large $(>3 \mathrm{~cm})$ and locally-advanced breast cancers (T3, T4, or N2) in order to reduce the size of the primary tumor and increase the likelihood of breast conservation, and to eliminate occult systemic metastases in order to improve survival. Tumor samples were acquired following $2-4$ cycles of TEC therapy $(n=3)$ and from patients who did not receive chemotherapy prior to surgery $(n=6)$. The resected primary tumor tissues were kept in liquid nitrogen following surgery. The response of primary tumors to TEC treatment was quantified according to the RECIST criteria (Response Evaluation Criteria in Solid Tumors).

Blood samples for assessing the response to chemotherapy following surgery: Peripheral blood samples were collected into polypropylene tubes containing EDTA (Vacutainer System, BD Biosciences, San Jose, CA, USA). The samples consisted of 2 groups: i) Patients who received 3-6 cycles of TEC therapy following surgery, extracted before the final cycle $(n=34)$; ii) Samples extracted when the pathological diagnosis of the patients was made at surgery $(n=21)$. The patient demographics and clinical pathological characteristics are listed in the Table I. Tumor assessment was performed by MRI and/ or ultrasound depending on the method used at baseline, and the response to chemotherapy was assessed by the RECIST criteria (13). Patients achieving a complete (CR) or partial (PR) response were considered to be responders; those with stable disease (SD) or progressive disease (PD) were considered to be non-responders. The use of clinical samples in this study was approved by the Review Board at the Fourth Affiliated Hospital of Soochow University.

Isolation of microvesicles. The methods for collecting isolated EVs from plasma were as previously described. The plasma supernatant was obtained by centrifugation at $850 \mathrm{x} \mathrm{g}$ for 3 min. The EVs were isolated after 1:2 dilution in PBS by sequential centrifugation $(10 \mathrm{~min}$ at $500 \mathrm{x}$ g to remove cell debris; $30 \mathrm{~min}$ at 2,000 x g to remove platelets; and $70 \mathrm{~min}$ at $100,000 \times \mathrm{g}$ to obtain precipitate). After washing in PBS, EVs were re-suspended in PBS for analysis.

Reverse transcriptase-polymerase chain reaction (RT-PCR). Isolated EVs were lysed with $200 \mu \mathrm{l}$ TRIzol reagent
(Invitrogen Life Technologies, Carlsbad, CA, USA) and processed according to the manufacturer's instructions to obtain total RNA. Total RNA was reverse-transcribed using a Reverse Transcriptase M-MLV (RNase H-) kit (Takara Bio Inc., Otsu, Japan) according to the manufacturer's instructions. The single-stranded cDNA was amplified by PCR in a Thermal Cycler C1000 Tech (Bio-Rad) using DreamTaq Green PCR Master Mix (2x) (Thermo-Scientific). GAPDH was used as an endogenous control. The PCR was performed under the following conditions: $3 \mathrm{~min}$ at $95^{\circ} \mathrm{C} ; 30 \mathrm{sec}$ at $94^{\circ} \mathrm{C}, 30 \mathrm{sec}$ at $58^{\circ} \mathrm{C}$, and $45 \mathrm{sec}$ at $72^{\circ} \mathrm{C}$ for 35 cycles; and $72^{\circ} \mathrm{C}$ for $10 \mathrm{~min}$. The primer sequences were as follows: BCRP, F 5'-CAGCCG TGGAACTCTTTGTGGTAGAGAAG-3' and R 5'-CTGTTG CATTGAGTCCTGGGCAGAAG-3'; flotillin-2, F 5'-AGA TCCGGCAGGAAGAGATT-3' and R 5'-GCTTCTGCCTTG AGCTTCAT-3'; MUC1, F 5'-CGACTACTACCAAGAGCT GCAGAGAGACAT-3' and R 5'-TGTAAGAGAGGCTGC TGCCACCATTACCTG-3'; GAPDH, F 5'-CTCCTGCAC CACCAACTGCTTAGC-3' and R 5'-CGCCTGCTTCACCAC CTTCTTGAT-3'. Equal amounts of RT-PCR products were loaded onto $1.5 \%$ agarose gels. The data were analyzed with ImageJ software (v.149; NIH, Bethesda, MD, USA). All reactions were performed in triplicate.

Immunofluorescence (IF) analysis. IF staining was performed as described previously (10). Isolated EVs were resuspended in PBS, and then passed through a $0.8-\mu \mathrm{m}$ filter (Stericup, Merck Millipore, Billerica, MA, USA), allowing EVs of diameters $<0.8 \mu \mathrm{m}$ to remain in the filtrate. The filtrate was then passed through a $0.1-\mu \mathrm{m}$ filter (Stericup), so purified EVs with diameters from 0.1 to $0.8 \mu \mathrm{m}$ were retained on the filter surface. The EVs on the filter underwent IF staining. The purified EVs and surgical specimens were fixed with $4 \%$ paraformaldehyde (Sigma-Aldrich, St. Louis, MO, USA) for $15 \mathrm{~min}$, then blocked with $10 \%$ BSA/PBS with $0.1 \%$ Triton X-100 (Bio-Rad Laboratories, Inc., Hercules, CA, USA) in PBS for $30 \mathrm{~min}$ at room temperature. Slides were incubated with primary antibodies overnight at $4{ }^{\circ} \mathrm{C}$ followed by the appropriate secondary fluorescence-labeled antibody for $1 \mathrm{~h}$ at room temperature. DAPI (1:1,000; Beyotime Institute of Biotechnology, Haimen, China) was used to stained nuclei. Images were captured with a confocal microscope (Leica TCS SP8, Leica Microsystems $\mathrm{GmbH}$, Wetzlar, Germany). The primary antibody mouse anti-BCRP/ABCG2 monoclonal antibody (Abcam, Cambridge, UK; ab3380, diluted 1:10) was used for IF staining in frozen sections of surgical specimens; mouse anti-BCRP/ABCG2 monoclonal antibody (Abcam, ab3380, diluted 1:10) and goat anti-MUC1 monoclonal antibody (Santa Cruz Biotechnology, Inc., Dallas, TX, USA; sc-6825, diluted 1:20) were used for IF staining of purified EVs. The secondary antibodies were Alexa Fluor 488-labeled donkey anti-mouse $\mathrm{IgG}(\mathrm{H}+\mathrm{L})$ antibody and Alexa Fluor 546-labeled donkey anti-goat IgG (H+L) antibody (Invitrogen Life Technologies; diluted 1:100).

Fluorescent in situ hybridization (FISH) analysis. FISH was performed using the locked nucleic acid (LNA)-modified oligonucleotide probe (Redlandbio Technology Co., Ltd., Guangzhou, China). The sequences of the hBCRP/ABCG2 probes were as follows: hBCRP probe 1, 5'-ATGCTGCAA AGCCGTAAATCCATATCGTG-3'; hBCRP probe 2, 5'-TAA 
A

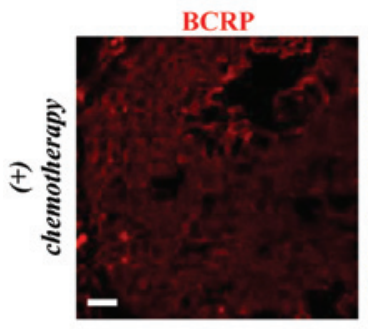

B

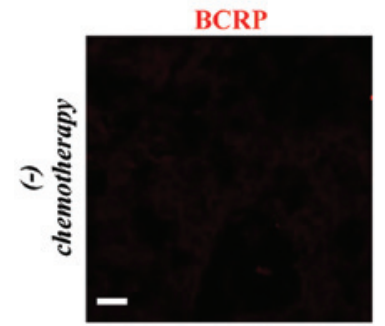

DAPI

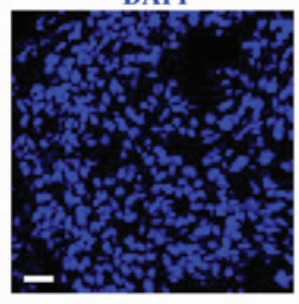

DAPI

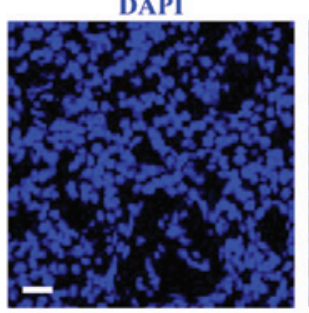

BCRP/DAPI

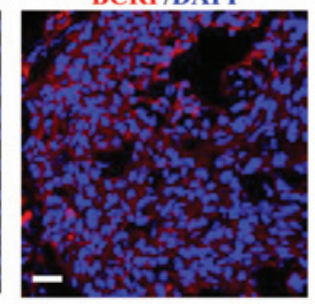

BCRP/DAPI

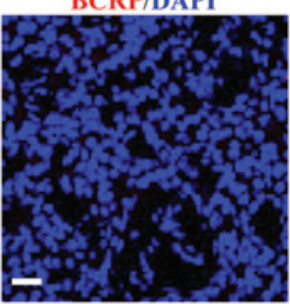

Figure 1. BCRP mRNA level is up-regulated in breast cancer with neoadjuvant chemotherapy. Representative images from FISH for BCRP mRNA in breast cancer specimens (A) with and (B) without chemotherapy; (scale bars, $25 \mu \mathrm{m}$ ). BRCP, breast cancer resistance protein; FISH, fluorescence in situ hybridization.

GATGACACTCTGTAGTATCCGCTGATG-3'; and hBCRP probe 3, 5'-CTCTACTCTACCCACAGTTCCAAACCC TCA-3'. Surgical specimens were washed with $0.1 \mathrm{mM}$ PBS, permeabilized with $0.4 \%$ Triton $\mathrm{X}-100$, and treated with $1 \mu \mathrm{g} / \mathrm{ml}$ proteinase $\mathrm{K}$ (Beyotime Institute of Biotechnology). The specimens were washed with 4\% PFA for $5 \mathrm{~min}$ to inhibit the effects of the proteinase. To reduce non-specific signals, slides were washed once with $0.25 \%$ acetic anhydride (Sigma-Aldrich). Hybridization with the probe $(1 \mu \mathrm{M})$ was performed at $40^{\circ} \mathrm{C}$ for $16 \mathrm{~h}$ after incubation in $50 \%$ formamide (Sangon Biotech Co., Ltd., Shanghai, China) that had been deionized for $30 \mathrm{~min}$ at $37^{\circ} \mathrm{C}$. The slides were washed once with $4 \mathrm{X}$ saline-sodium citrate (SSC) for $15 \mathrm{~min}$ at $37^{\circ} \mathrm{C}$, and once with NTE buffer $(500 \mathrm{mM} \mathrm{NaCl}, 10 \mathrm{mM}$ Tris, $1 \mathrm{mM}$ EDTA, $20 \mu \mathrm{g} / \mathrm{ml}$ RNase A) for $30 \mathrm{~min}$ at $37^{\circ} \mathrm{C}$ to digest the single-stranded RNA, then rinsed once with $1 \mathrm{X} \mathrm{SSC}$ and $0.5 \mathrm{X} \mathrm{SSC}\left(15 \mathrm{~min}\right.$ each) at $37^{\circ} \mathrm{C}$, and finally washed twice with Buffer $1(100 \mathrm{mM}$ Tris, $150 \mathrm{mM} \mathrm{NaCl}$, $\mathrm{pH}$ 7.5) for $10 \mathrm{~min}$. All FISH images of BCRP were captured by a confocal laser scanning microscope (Leica TCS SP8, Leica Microsystems GmbH).

Flow cytometry. Analysis of purified EVs. Purified EVs $[10 \mu \mathrm{g}$ as measured by Bradford assay (Sangon Biotech Co., Ltd.)] were incubated with $10 \mu \mathrm{l}$ latex beads $3.1 \mu \mathrm{m}$ in diameter (Life Technologies, Gaithersberg, MD, USA) in a $1.5-\mathrm{ml}$ microcentrifuge tube for $15 \mathrm{~min}$ at room temperature. PBS was added to a final volume of $1 \mathrm{ml}$ and incubated on a test-tube rotator overnight at $4^{\circ} \mathrm{C}$. Glycine was added $(110 \mu \mathrm{l}$ of $1 \mathrm{M} ; 100 \mathrm{mM}$ final concentration), mixed gently, incubated at room temperature for $30 \mathrm{~min}$, and then centrifuged for $3 \mathrm{~min}$ at 1,500 $\mathrm{xg}$ at room temperature. The supernatant was removed and discarded. The bead pellet was re-suspended in $1 \mathrm{ml} \mathrm{PBS} / 0.5 \%$ BSA and centrifuged for $3 \mathrm{~min}$ at $1,500 \mathrm{x} \mathrm{g}$ at room temperature. The supernatant was removed and discarded, and the pellet washed twice. EV-coated beads were incubated for $1 \mathrm{~h}$ at room temperature in $50 \mu \mathrm{lPSB} / 5 \% \mathrm{BSA}$ containing the primary antibody, either mouse anti-BCRP/ ABCG2 monoclonal antibody (Abcam; ab3380, diluted 1:10), mouse anti-MUC1 monoclonal antibody (Abcam; ab70475, diluted 1:50), mouse anti-flotillin-2 monoclonal antibody (Santa Cruz Biotechnology; sc-28320, diluted 1:50), or mouse isotype-matched control IgG1 (Abcam: ab18443, diluted 1:100). The pellet was resuspended in $1 \mathrm{ml} \mathrm{PBS} / 0.5 \%$ BSA and centrifuged for $3 \mathrm{~min}$ at $1,500 \mathrm{xg}$ at room temperature. The secondary antibody Alexa Fluor 488-labeled donkey anti-mouse $\operatorname{IgG}(\mathrm{H}+\mathrm{L})$ (Invitrogen Life Technologies, diluted 1:100) was incubated for $45 \mathrm{~min}$ at room temperature, followed by washing and resuspension with PBS. The antibody-stained EV-coated latex beads were analyzed on a FACSCalibur flow cytometer (BD Biosciences, Mountain View, CA, USA). Data were analyzed using Flowjo software, version 7.6 (Flowjo, LLC, Ashland, OR, USA).

Statistical analysis. Results are presented as the mean \pm standard error of the mean. All experiments were performed in triplicate. Statistical differences were determined by the paired Student's $t$-test, using Graphpad Prism software, version 5.0 (GraphPad Software, Inc., La Jolla, CA, USA). All statistical tests were two-sided, and $\mathrm{P}<0.05$ was considered to indicate a statistically significant difference.

\section{Results}

BCRP expression is upregulated at the $m R N A$ level in tumor specimens from patients who received neoadjuvant chemotherapy prior to surgery. A total of 3 tumor specimens were collected from each patient who received TEC therapy and was assessed as non-responsive ( $\mathrm{PD} / \mathrm{SD}$ according to the RECIST criteria) and those who received no chemotherapy. FISH analysis demonstrated that the tumor samples from non-responsive patients had higher mRNA expression levels of BCRP compared with the group with no chemotherapy (Fig. 1), indicating a potential role of BCRP in drug resistance.

BCRP and flotillin-2 expression in tumor specimens is enhanced following neoadjuvant chemotherapy. IF was used to assess the expression of BCRP and the EV marker flotillin-2 in specimens 
Table I. Clinical and pathological characteristics of 34 patients who received chemotherapy and 21 patients who did not receive chemotherapy after surgery (blood drawn at the time of primary diagnosis of breast cancer).

\begin{tabular}{|c|c|c|c|}
\hline Characteristics & $\begin{array}{l}\text { All patients } \\
(\mathrm{n}=55)\end{array}$ & $\begin{array}{c}+ \text { Chemotherapy } \\
(\mathrm{n}=34)\end{array}$ & $\begin{array}{l}\text {-Chemotherapy } \\
\quad(\mathrm{n}=21)\end{array}$ \\
\hline \multicolumn{4}{|l|}{ Age, years } \\
\hline$<50$ & 15 & 10 & 5 \\
\hline$\geq 50$ & 40 & 24 & 16 \\
\hline \multicolumn{4}{|l|}{ Gender } \\
\hline Male & 0 & 0 & 0 \\
\hline Female & 55 & 34 & 21 \\
\hline \multicolumn{4}{|l|}{ Histology } \\
\hline Ductal & 53 & 32 & 21 \\
\hline Paget disease & 2 & 2 & 0 \\
\hline \multicolumn{4}{|l|}{ Tumor size } \\
\hline pTx & 3 & 3 & 0 \\
\hline pT1 & 11 & 7 & 4 \\
\hline pT2 & 26 & 12 & 14 \\
\hline pT3 & 9 & 7 & 2 \\
\hline pT4 & 6 & 5 & 1 \\
\hline \multicolumn{4}{|l|}{ Lymph node status } \\
\hline N0 & 21 & 8 & 13 \\
\hline N1 & 15 & 10 & 5 \\
\hline $\mathrm{N} 2$ & 9 & 8 & 1 \\
\hline N3 & 10 & 8 & 2 \\
\hline \multicolumn{4}{|l|}{ AJCC Substage } \\
\hline I & 6 & 4 & 2 \\
\hline II & 28 & 13 & 15 \\
\hline III & 13 & 9 & 4 \\
\hline IV & 8 & 8 & 0 \\
\hline \multicolumn{4}{|l|}{ Adjuvant Chemotherapy } \\
\hline Anthracycline & 10 & 10 & 0 \\
\hline Taxane & 9 & 9 & 0 \\
\hline Anthracycline/taxane & 15 & 15 & 0 \\
\hline \multicolumn{4}{|l|}{ ER Status } \\
\hline Positive & 38 & 22 & 16 \\
\hline Negative & 15 & 10 & 5 \\
\hline Missing & 2 & 2 & 0 \\
\hline \multicolumn{4}{|l|}{ PR Status } \\
\hline Positive & 37 & 21 & 16 \\
\hline Negative & 15 & 10 & 5 \\
\hline Missing & 3 & 3 & 0 \\
\hline \multicolumn{4}{|l|}{ Her-2 Status } \\
\hline Positive & 31 & 18 & 13 \\
\hline Negative & 16 & 8 & 8 \\
\hline Missing & 8 & 8 & 0 \\
\hline
\end{tabular}

AJCC, Americal Joint Committee of Cancer; ER, estrogen receptor; PR, progesterone receptor; Her-2, human epidermal growth factor receptor 2 .

from non-responsive patients and those without chemotherapy by IF. The results demonstrated that both BCRP and flotillin-2 were expressed at higher levels in samples from non-responsive patients compared with controls (Fig. 2A and B). These results indicated that BCRP and flotillin-2 may be potentially used as indicators of the response to neoadjuvant chemotherapy. 
A

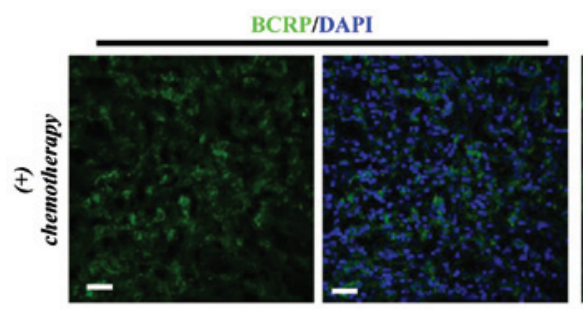

B

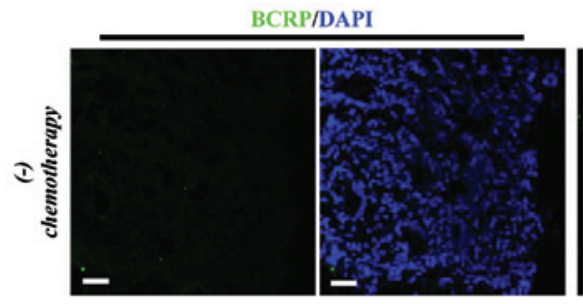

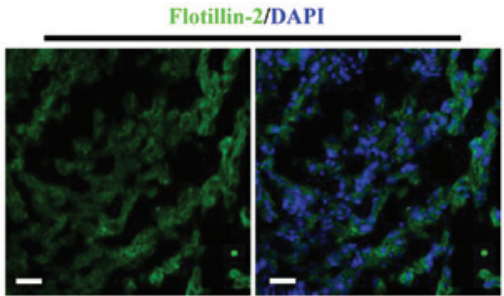

Flotillin-2/DAPI

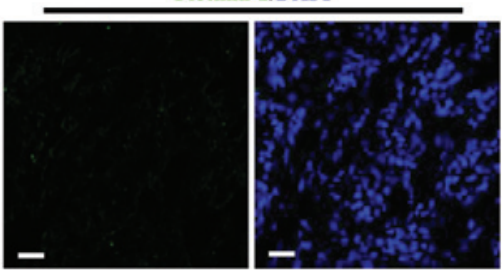

Figure 2. Both BCRP and flotillin-2 expression of breast cancer specimens is enhanced by neoadjuvant chemotherapy. Confocal micrographs showing immunofluorescence staining for BCRP and flotillin-2 in breast cancer specimens (A) with and (B) without chemotherapy (scale bars, $25 \mu \mathrm{m}$ ). BRCP, breast cancer resistance protein.
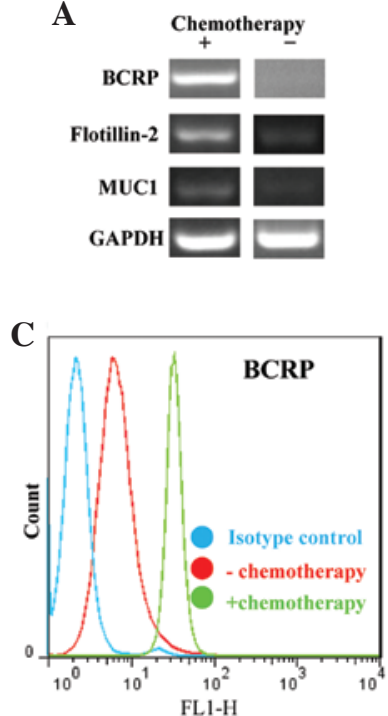

\section{B}
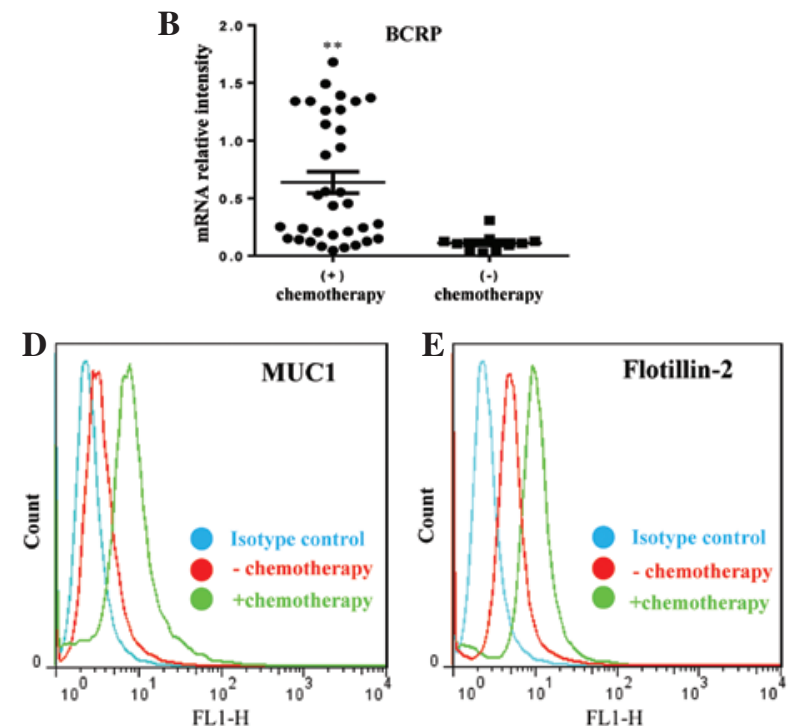

Figure 3. Circulating EVs have higher BCRP expression at the mRNA and protein levels in non-responsive patients than those without chemotherapy after surgery. (A) Representative images of the gel and (B) quantification of reverse transcription-polymerase chain reaction results demonstrating upregulation of three pooled transcripts (BCRP, flotillin-2, and MUC1) in peripheral blood EVs from non-responsive patients. FACS assays showing notable shifts of (C) BCRP, (D) MUC1, and (E) flotillin-2 immunofluorescence in circulating EVs from non-responsive patients. ${ }^{* *} \mathrm{P}<0.01$ vs. (-) chemotherapy (Student's $t$-test). $\mathrm{BRCP}$, breast cancer resistance protein; MUC1, mucin 1; EVs, extracellular vesicle.

Patients with PD/SD (non-responders) demonstrate higher $B C R P$ expression levels in circulating EVs. As noted above, both BCRP and flotillin-2 were upregulated in tumor specimens from non-responsive patients. Since flotillin-2 is an EV marker, to further study the association between BCRP and EVs, the BCRP levels were assessed in circulating EVs isolated from patients at the mRNA and protein levels using RT-PCR and FACS, respectively. It is known that MUC1 is frequently expressed in breast cancer, is sorted into rafts by a flotillin-2-dependent mechanism, and is exported via EVs (14-16). Therefore, RT-PCR was performed to simultaneously identify the transcript expression of BCRP, flotillin2, and MUC1. As hypothesized, these transcripts were expressed at significantly higher levels in blood samples from non-responsive patients compared with those without chemotherapy after surgery (Fig. 3A). Samples were obtained from 34 patients who received 4-6 cycles of TEC therapy and 21 patients without chemotherapy (Table I). Analysis of BCRP transcription levels (Fig. 3B) demonstrated that BCRP transcription was only detectable in the circulating EVs of 10/21 (47.6\%) of patients without chemotherapy; in $11 / 21$ patients without chemotherapy it was too low to be detected. The FACS results were in accordance with the RT-PCR by demonstrating increased expression levels of BCRP, flotillin-2, and MUC1 in EVs from non-responsive patients (Fig. 3C-E). Taken together, circulating EVs containing BCRP of tumor origin may be potentially used as a prognostic biomarker for the response to clinical chemotherapy.

Tumor-derived circulating EVs expressing BCRP demonstrate notable potential for predicting chemotherapeutic outcome. To present the association between BCRP and MUC1 visually, 
A

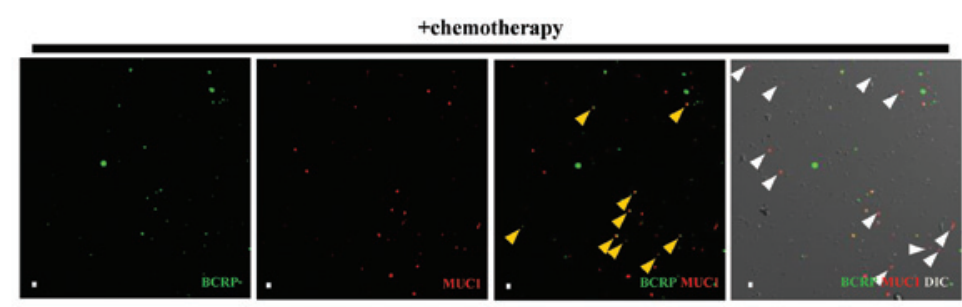

$\mathbf{B}$
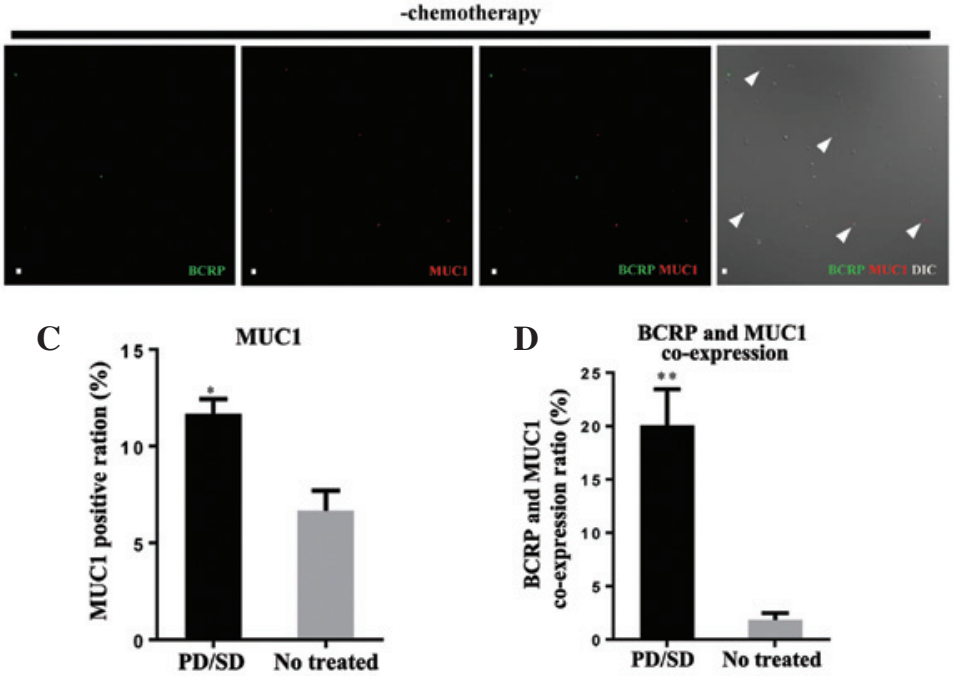

Figure 4. Tumor-derived circulating EVs expressing BCRP are up-regulated in non-responsive patients. Representative images of immunofluorescence staining showing significantly elevated (A) BCRP and (B) MUC1 expression in EVs from non-responsive patients (A) compared to those with no chemotherapy (B). White arrows, MUC1-positive signals; yellow arrows, co-expression of MUC1 and BCRP signals. (C) Summary data showing that the MUC1-positive EV ratio to total EVs from non-responsive patients was higher than that in patients without chemotherapy. (D) Summary data showing the ratio of circulating EVs that co-expressed MUC1 with BCRP to the total MUC1-positive EVs from non-responsive patients and those without chemotherapy ( ${ }^{*}<0.05$, ${ }^{* *} \mathrm{P}<0.01$ vs. untreated (Student's $t$-test); scale bars, $1 \mu \mathrm{m}$ ). BRCP, breast cancer resistance protein; MUC1, mucin 1; EVs, extracellular vesicle; PD, progressive disease; SD, stable disease.

immunostaining was used with specific anti-BCRP/ABCG2 and anti-MUC1 monoclonal antibodies to visualize their expression in EVs from the peripheral blood of patients. The results demonstrated that the EVs from non-responsive patients had higher MUC1 expression (Fig. 4A-C), indicating their higher release of EVs into the circulation. Notably, BCRP was co-expressed with MUC1 in samples from non-responsive patients, however this was almost absent from the control group (Fig. 4 A, B and D), which indicates that the development of chemoresistance may be due to a progressive enrichment of BCRP in EVs and their release. All these findings support the idea that tumor-derived circulating EVs that carry BCRP could be used as a prognostic biomarker to predict the chemotherapeutic outcome in breast cancer.

\section{Discussion}

BCRP can confer a multidrug-resistant phenotype on cancer cells and affects drug absorption, distribution, metabolism, and excretion in normal tissues (11), however, the underlying mechanism remains unknown. To the best of our knowledge, this is the first study to investigate the association between BCRP and circulating EVs, elucidating the mechanism of clinical drug resistance.

Previous studies have demonstrated that recipient cells can acquire drug resistance by the transmission of P-glycoprotein or $\mathrm{Ca}^{2+}$-permeable channels via EVs (10). The association between
BCRP-containing EVs and poor outcomes of clinical chemotherapy were further verified. In the present study, higher BCRP expression at both the mRNA and proteins levels was observed in tumor specimens and EVs in blood samples from patients with a poor chemotherapeutic outcome (non-responders) compared with those with no chemotherapy. It has previously been reported that flotillin-2 overexpression is associated with a poor prognosis and reduced survival in patients with both early- and late-stage breast cancer (17). Moreover, Ma et al (10) demonstrated that flotillin-2 expression is significantly upregulated following chemotherapy and the present study confirmed this. Based on these findings, BCRP and flotillin-2 may be indicators of the potential response to neoadjuvant chemotherapy. Moreover, the co-expression level of MUC1 and BCRP also demonstrated a close associationwith the outcome of chemotherapy.

It has been reported that, apart from BCRP, the expression levels of MDR2, LRP, and MRP1 all have predictive value for the clinical outcome of adjuvant chemotherapy (18). The present study indicated that a high level of BCRP co-expression with MUC1 was induced by TEC therapy but the underlying mechanism needs to be further defined. To assess the clinical response to chemotherapy, blood tumor markers such as CA15-3, carcinoembryonic antigen, and erythrocyte sedimentation rate are usually used as treatment guidelines (19). However, they do not reflect accurately the status of patients in response to chemotherapy. Therefore, it is important to identify more appropriate biomarkers to predict the chemotherapeutic response. In the 
present study, significantly different levels of BCRP-containing EVs were observed between patients with or without chemoresistance, and the novel hypothesis that the level of tumor-derived circulating EVs in which BCRP is expressed can predict the chemotherapeutic response was proposed, which provides a convenient means of determining an individual therapeutic strategy when facing the challenge of drug resistance.

Nevertheless, questions remain, such as: i) The exact level at which BCRP-containing EVs determine non-responsiveness. ii) Whether the underlying mechanism of BCRP enrichment in circulating EVs is intrinsic or acquired. iii) Whether specific chemotherapy drugs have specific relationships with EVs. iv) Whether the TNM stage can be determined by measuring the level of BCRP-containing circulating EVs. v) Whether estrogen receptor/progesterone receptor/human epidermal growth factor receptor 2 status or overall/progression-free survival can be determined by EV measurement. Therefore, further exploration of these problems may be worthwhile for tapping the potential of BCRP-containing EVs as a predictor of chemotherapeutic outcome.

\section{Acknowledgements}

The authors would like to thank Prof. Iain C. Bruce for critical reading of the manuscript. The present study was supported by the Major Research plan of the National Natural Science Foundation of China (grant no. 91439131 to Dr Xin Ma); the Natural Science Foundation for Distinguished Young Scholars of Jiangsu Province (grant no. BK20140004 to Dr Xin Ma); the Program for New Century Excellent Talents in University of The Ministry of Education of China (Grant no. NCET-12-0880 to Dr Xin Ma); Fundamental Research Funds for the Central Universities (grant no's. JUSRP51311A and JUSRP51516 to Dr Xin Ma); China National Natural Science Foundation grants (grant no. 81100185 to Dr Xin Ma, 81273437 and 31200126 to Dr Jian Jin); and an NSFC-RGC joint grant (grant no. 81361168001 to Dr Jian Jin). The Clinical Science and Technology Projects (grant no. BL2014019 to Dr Dong Hua).

\section{References}

1. Holohan C, Van Schaeybroeck S, Longley DB and Johnston PG: Cancer drug resistance: An evolving paradigm. Nat Rev Cancer 13: 714-726, 2013.

2. Cheung KL, Evans AJ and Robertson JF: The use of blood tumour markers in the monitoring of metastatic breast cancer unassessable for response to systemic therapy. Breast Cancer Res Treat 67: 273-278, 2001.
3. Weigel MT and Dowsett M: Current and emerging biomarkers in breast cancer: Prognosis and prediction. Endocr Relat Cancer 17: R245-R262, 2010.

4. Raposo G and Stoorvogel W: Extracellular vesicles: Exosomes, microvesicles and friends. J Cell Biol 200: 373-383, 2013.

5. Holme PA, Solum NO, Brosstad F, Røger M and Abdelnoor M: Demonstration of platelet-derived microvesicles in blood from patients with activated coagulation and fibrinolysis using a filtration technique and western blotting. Thromb Haemost 72: 666-671, 1994.

6. Hess C, Sadallah S, Hefti A, Landmann R and Schifferli JA: Ectosomes released by human neutrophils are specialized functional units. J. Immunol 163: 4564-4573, 1999.

7. Cocucci E, Racchetti G and Meldolesi J: Shedding microvesicles: Artefacts no more. Trends Cell Biol 19: 43-51, 2009.

8. György B, Szabó TG, Pásztói M, Pál Z, Misják P, Aradi B, László V, Pállinger E, Pap E, Kittel A, et al: Membrane vesicles, current state-of-the-art: Emerging role of extracellular vesicles. Cell Mol Life Sci 68: 2667-2688, 2011.

9. Harding CV, Heuser JE and Stahl PD: Exosomes: Looking back three decades and into the future. J Cell Biol 200: 367-371, 2013.

10. Ma X, Chen Z, Hua D, He D, Wang L, Zhang P, J, Cai Y, Gao C, Zhang X, et al: Essential role for TrpC5-containing extracellular vesicles in breast cancer with chemotherapeutic resistance. Proc Natl Acad Sci USA 111: 6389-6394, 2014.

11. Noguchi K, Katayama K and Sugimoto Y: Human ABC transporter ABCG2/BCRP expression in chemoresistance: Basic and clinical perspectives for molecular cancer therapeutics. Pharmgenomics Pers Med 7: 53-64, 2014.

12. Brangi M, Litman T, Ciotti M, Nishiyama K, Kohlhagen G, Takimoto C, Robey R, Pommier Y, Fojo T and Bates SE: Camptothecin resistance: Role of the ATP-binding cassette (ABC), mitoxantrone-resistance half-transporter (MXR), and potential for glucuronidation in MXR-expressing cells. Cancer Res 59: 5938-5946, 1999.

13. Eisenhauer EA, Therasse P, Bogaerts J, et al: New response evaluation criteria in solid tumours: Revised RECIST guideline (version 1.1). Eur J Cancer 45: 228-247, 2009.

14. Staubach S, Razawi $\mathrm{H}$ and Hanisch FG: Proteomics of MUC1-containing lipid rafts from plasma membranes and exosomes of human breast carcinoma cells MCF-7. Proteomics 9: 2820-2835, 2009.

15. Brouckaert O, Laenen A, Wildiers H, Floris G, Moerman P, Van Limbergen E, Vergote I, Billen J, Christiaens MR and Neven P: The prognostic role of preoperative and (early) postoperatively change in CA15.3 serum levels in a single hospital cohort of primary operable breast cancers. Breast 22: 254-262, 2013.

16. Gion M, Mione R, Leon AE and Dittadi R: Comparison of the diagnostic accuracy of CA27.29 and CA15.3 in primary breast cancer. Clin Chem 45: 630-637, 1999.

17. Wang X, Yang Q, Guo L, et al: Flotillin-2 is associated with breast cancer progression and poor survival outcomes. J Transl Med 11: 190, 2013.

18. Burger H, Foekens JA, Look MP, Meijer-van Gelder ME, Klijn JG, Wiemer EA, Stoter G and Nooter K: RNA expression of breast cancer resistance protein, lung resistance-related protein, multidrug resistance-associated proteins 1 and 2 and multidrug resistance gene 1 in breast cancer: correlation with chemotherapeutic response. Clin Cancer Res 9: 827-836, 2003.

19. Marić P, Ozretić P, Levanat S, Oresković S, Antunac K and Beketić-Oresković: Tumor markers in breast cancer - evaluation of their clinical usefulness. Coll Antropol 35: 241-247, 2011. 